\title{
On the Pasting Lemma on a Fuzzy Soft Topological Space with Mixed Structure
}

\author{
Nihal Taş*
}

\begin{abstract}
In this paper, we define the notion of a $\left(v_{1}, v_{2}\right)$-generalized closed fuzzy soft set (shorlty, a $\left(v_{1}, v_{2}\right)$-g-closed fuzzy soft set) on a fuzzy soft topological space. Using this notion, we investigate some properties of a $\left(v_{1}, v_{2}\right)$-g-closed fuzzy soft set and prove a new version of the "Pasting Lemma" with a mixed structure.

Keywords: Pasting lemma; Fuzzy soft topological space; $\left(v_{1}, v_{2}\right)$-g-closed fuzzy soft set; Mixed structure.

AMS Subject Classification (2020): Primary: 54A40 ; Secondary: 06D72; 54C10; 54A05.

${ }^{*}$ Corresponding author
\end{abstract}

\section{Introduction and Motivation}

Soft set theory was introduced as a new approach to the mathematical tool for coping with encountered problems in different sciences [18]. After then, in [17], the notion of a fuzzy soft set was presented combined with the notions of a soft set [18] and a fuzzy set [28] as a generalization of fuzzy set theory. Using this new theory, some topological concepts and their basic properties were studied via different approaches (for example, see $[3,8,9,16,17,20,22,26,27]$ and the references therein). On the other hand, some applications of the fuzzy soft set theory were given to other sciences as an another important approach (see [4, 10, 12, 13, 15, 21, 24]).

Recently, some topological notions and properties have been generalized using the mixed structure on various generalized topological spaces such as soft topological space, generalized topological space etc. (see $[1,2,7,23])$. One of the important topological notions is the pasting lemma for continuous functions. For example, this notion has an important role in algebraic topology. Many researchers have studied on various versions of the pasting lemma with different aspects (see $[5,6,11,14,25]$ and the references therein for more details).

In this paper, we focus on the pasting lemma on a fuzzy soft topological space for mixed $g$-fuzzy soft continuous functions. To do this, we define the concept of a $\left(v_{1}, v_{2}\right)$-g-closed fuzzy soft set with a mixed structure. We investigate some properties of this new notion with some necessary examples. Then using the notion of a $\left(v_{1}, v_{2}\right)-g$ closed fuzzy soft set, we introduce the mixed $g$-fuzzy soft continuity on a fuzzy soft topological space. Finally, we prove a new version of the pasting lemma with regard to the mixed $g$-fuzzy soft continuity.

\section{Preliminaries}

In this section, we recall some basic concepts related to fuzzy soft set theory. Throughout this paper, we assume that $U$ is an initial universal set, $E$ is a nonempty set of parameters and $A, B \subseteq E$.

Definition 2.1. [28] A fuzzy set $F$ on $U$ is a mapping $F: U \rightarrow I$. The value $F(u)$ represents the degree of membership of $u \in U$ in the fuzzy set $F$ for $u \in U$.

Let $I^{U}$ denotes the family of all fuzzy sets on $U$. If $F, G \in I^{U}$ then some basic set operations are given in [28] as follows:

(i) $F \leq G \Leftrightarrow F(u) \leq G(u)$ for all $u \in U$.

Received : 15-11-2019, Accepted : 22-06-2020 
(ii) $F=G \Leftrightarrow F(u)=G(u)$ for all $u \in U$.

(iii) $H=F \vee G \Leftrightarrow H(u)=F(u) \vee G(u)$ for all $u \in U$.

(iv) $K=F \wedge G \Leftrightarrow K(u)=F(u) \wedge G(u)$ for all $u \in U$.

(v) $C=F^{c} \Leftrightarrow C(u)=1-F(u)$ for all $u \in U$.

Definition 2.2. [17] A pair $(\alpha, A)$, denoted by $\alpha_{A}$, is called a fuzzy soft set over $U$, where $\alpha: A \rightarrow I^{U}$ is a function. The family of all fuzzy soft sets over $U$ denoted by $F S S(U)_{E}$.

Definition 2.3. $[3,17,27]$ Let $\alpha_{A}, \beta_{A} \in F S S(U)_{E}$. Then the followings hold:

(i) The fuzzy soft set $\alpha_{A}$ is called a null fuzzy soft set if $\alpha(e)=0_{U}$ for each $e \in A$. It is denoted by $\widetilde{\emptyset}$.

(ii) The fuzzy soft set $\alpha_{A}$ is called an absolute fuzzy soft set if $\alpha(e)=1_{U}$ for each $e \in A$. It is denoted by $\widetilde{U}$.

(iii) $\alpha_{A}$ is a fuzzy soft subset of $\beta_{A}$ if $\alpha(e) \leq \beta(e)$ for each $e \in A$. It is denoted by $\alpha_{A} \sqsubseteq \beta_{A}$.

(iv) $\alpha_{A}$ and $\beta_{A}$ are equal if $\alpha_{A} \sqsubseteq \beta_{A}$ and $\beta_{A} \sqsubseteq \alpha_{A}$. It is denoted by $\alpha_{A}=\beta_{A}$.

$(v)$ The complement of a fuzzy soft set $\alpha_{A}$ is denoted by $\alpha_{A}^{c}$, where $\alpha^{c}: A \rightarrow I^{U}$ is a mapping defined by

$$
\alpha^{c}(e)=1_{U}-\alpha(e)
$$

for all $e \in A$. Also, we have $\left(\alpha_{A}^{c}\right)^{c}=\alpha_{A}$.

(vi) The union of $\alpha_{A}$ and $\beta_{A}$ is a fuzzy soft set $\gamma_{A}$ defined by

$$
\gamma(e)=\alpha(e) \vee \beta(e),
$$

for all $e \in A$. It is denoted by $\gamma_{A}=\alpha_{A} \sqcup \beta_{A}$.

(vii) The intersection of $\alpha_{A}$ and $\beta_{A}$ is a fuzzy soft set $\gamma_{A}$ defined by

$$
\gamma(e)=\alpha(e) \wedge \beta(e)
$$

for all $e \in A$. It is denoted by $\gamma_{A}=\alpha_{A} \sqcap \beta_{A}$.

Definition 2.4. [3] Let $I$ be an arbitrary index set and $\left\{\alpha_{i_{A}}\right\}_{i \in I}$ be a family of fuzzy soft sets on $U$. Then we have

(i) The union of these fuzzy soft sets is the fuzzy soft set $\gamma_{A}$ defined by

$$
\gamma(e)=\vee_{i \in I} \alpha_{i}(e),
$$

for all $e \in A$. It is denoted by $\gamma_{A}=\sqcup_{i \in I} \alpha_{i_{A}}$.

(ii) The intersection of these fuzzy soft sets is the fuzzy soft set $\gamma_{A}$ defined by

$$
\gamma(e)=\wedge_{i \in I} \alpha_{i}(e),
$$

for all $e \in A$. It is denoted by $\gamma_{A}=\sqcap_{i \in I} \alpha_{i_{A}}$.

Definition 2.5. [22] Let $v$ be the collection of fuzzy soft sets on $U$. Then $v$ is called a fuzzy soft topology on $U$ if the following conditions hold:

$\left(F S-t_{1}\right) \widetilde{\emptyset}, \widetilde{U} \in v$,

$\left(F S-t_{2}\right)$ The union of any number of fuzzy soft sets in $v$ belongs to $v$,

$\left(F S-t_{3}\right)$ The intersection of any two fuzzy soft sets in $v$ belongs to $v$.

The pair $(U, v)$ is called a fuzzy soft topological space. The members of $v$ are called fuzzy soft open sets. Also a fuzzy soft set $\alpha_{A}$ is called a fuzzy soft closed set if $\alpha_{A}^{c} \in v$.

Definition 2.6. [27] Let $(U, v)$ be a fuzzy soft topological space and $\alpha_{A} \in F S S(U)_{E}$. The fuzzy soft closure of $\alpha_{A}$, denoted by $v-F c l\left(\alpha_{A}\right)$ (or $\left.F c l\left(\alpha_{A}\right), \overline{\alpha_{A}}\right)$, is the intersection of all fuzzy soft closed supersets of $\alpha_{A}$. We note that $v-F c l\left(\alpha_{A}\right)$ is the smallest fuzzy soft closed set over $U$ which contains $\alpha_{A}$ and $v-F c l\left(\alpha_{A}\right)$ is closed.

Theorem 2.1. [27] Let $(U, v)$ be a fuzzy soft topological space and $\alpha_{A}, \beta_{B} \in F S S(U)_{E}$. Then the followings hold:

(i) $v-F c l(\widetilde{\emptyset})=\widetilde{\emptyset}$ and $v-F c l(\widetilde{U})=\widetilde{U}$.

(ii) $\alpha_{A} \sqsubseteq v-F c l\left(\alpha_{A}\right)$.

(iii) $v-F c l\left(v-F c l\left(\alpha_{A}\right)\right)=v-F c l\left(\alpha_{A}\right)$.

(iv) If $\alpha_{A} \sqsubseteq \beta_{B}$ then $v-F c l\left(\alpha_{A}\right) \sqsubseteq v-F c l\left(\beta_{B}\right)$.

(v) $\alpha_{A}$ is a fuzzy soft closed set if and only if $\alpha_{A}=v-F c l\left(\alpha_{A}\right)$.

(vi) $v-F c l\left(\alpha_{A} \sqcup \beta_{B}\right)=v-F c l\left(\alpha_{A}\right) \sqcup v-F c l\left(\beta_{B}\right)$. 
Definition 2.7. [22] Let $(U, v)$ be a fuzzy soft topological space and $V \subseteq U$. Let $\gamma_{E}^{V}$ be a fuzzy soft set over $V$ such that $\gamma_{E}^{V}: E \rightarrow I^{V}$ defined as $\gamma_{E}^{V}(e)=\mu_{\gamma_{E}^{V}}^{e}$ with

$$
\mu_{\gamma_{E}^{V}}^{e}(u)= \begin{cases}1 & , \quad u \in V \\ 0, & u \notin V\end{cases}
$$

Let $v_{V}=\left\{\gamma_{E}^{V} \sqcap \beta_{B}: \beta_{B} \in v\right\}$, then the fuzzy soft topology $v_{V}$ on $V$ is called fuzzy soft subspace topology for $V$ and $\left(V, v_{V}\right)$ is called fuzzy soft subspace of $(U, v)$.

Theorem 2.2. [16] The fuzzy soft set $\gamma_{E}$ is fuzzy soft closed in a subspace $\left(\beta_{E}, v_{\beta_{E}}\right)$ of $\left(\alpha_{E}, v\right)$ if and only if $\gamma_{E}=\eta_{E} \sqcap \beta_{E}$ for some fuzzy soft closed set $\eta_{E}$ in $\alpha_{E}$.

Theorem 2.3. [16] The fuzzy soft closure of a fuzzy soft set $\gamma_{E}$ in a subspace $\left(\beta_{E}, v_{\beta_{E}}\right)$ of $\left(\alpha_{E}, v\right)$ equals $v-F c l\left(\gamma_{E}\right) \sqcap \beta_{E}$.

Definition 2.8. [27] Let $F S S(U)_{E}$ and $F S S(V)_{E^{\prime}}$ be families of fuzzy soft sets over $U$ and $V$, respectively. Let $u: U \rightarrow V$ and $p: E \rightarrow E^{\prime}$ be mappings. Then the map $f_{p u}$ is called a fuzzy soft mapping from $U$ to $V$ and denoted by $f_{p u}: F S S(U)_{E} \rightarrow F S S(V)_{E^{\prime}}$ such that

(i) If $\alpha_{A} \in F S S(U)_{E}$, then the image of $\alpha_{A}$ under the fuzzy soft mapping $f_{p u}$ is the fuzzy soft set over $V$ defined by $f_{p u}\left(\alpha_{A}\right)$, where

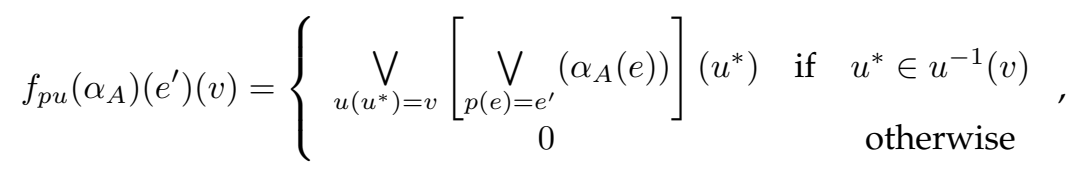

for all $e^{\prime} \in p(E)$ and all $v \in V$.

(ii) If $\beta_{B} \in F S S(V)_{E^{\prime}}$, then the pre-image of $\beta_{B}$ under the fuzzy soft mapping $f_{p u}$ is the fuzzy soft set over $U$ defined by $f_{p u}^{-1}\left(\beta_{B}\right)$, where

$$
f_{p u}^{-1}\left(\beta_{B}\right)(e)\left(u^{*}\right)=\left\{\begin{array}{ccc}
\beta_{B}(p(e))\left(u\left(u^{*}\right)\right) & \text { if } & p(e) \in B \\
0 & \text { otherwise }
\end{array},\right.
$$

for all $e \in p^{-1}\left(E^{\prime}\right)$ and all $u^{*} \in U$.

\section{A new version of the Pasting Lemma}

In this section, we present a new version of the pasting lemma on a fuzzy soft topological space using the notion of a $\left(v_{1}, v_{2}\right)$-g-closed fuzzy soft set. To do this, we begin the following definition.

Definition 3.1. Let $v_{1}, v_{2}$ be two fuzzy soft topologies on $U$ and $\alpha_{A} \in F S S(U)_{E}$. Then $\alpha_{A}$ is called a $\left(v_{1}, v_{2}\right)-g$ closed fuzzy soft set if $v_{2}-F c l\left(\alpha_{A}\right) \sqsubseteq \beta_{A}$ whenever $\alpha_{A} \sqsubseteq \beta_{A}$ and $\beta_{A}$ is a fuzzy soft open set according to $v_{1}$. The complement of a $\left(v_{1}, v_{2}\right)$-g-closed fuzzy soft set is called $\left(v_{1}, v_{2}\right)$-g-open fuzzy soft.

Example 3.1. Let $U=\left\{u_{1}, u_{2}, u_{3}\right\}, E=\left\{e_{1}, e_{2}\right\}, v_{1}=\left\{\widetilde{\emptyset}, \widetilde{U}, \alpha_{E}\right\}$ and $v_{2}=\{\widetilde{\emptyset}, \widetilde{U}\}$ where $\alpha_{E}$ is a fuzzy soft set on $U$ defined as

$$
\alpha_{E}=\left\{\left(e_{1},\left\{\frac{u_{1}}{0.4}, \frac{u_{2}}{0}, \frac{u_{3}}{0}\right\}\right),\left(e_{2},\left\{\frac{u_{1}}{0}, \frac{u_{2}}{0.3}, \frac{u_{3}}{0}\right\}\right)\right\} .
$$

Then the fuzzy soft set

$$
\beta_{E}=\left\{\left(e_{1},\left\{\frac{u_{1}}{0.4}, \frac{u_{2}}{0.2}, \frac{u_{3}}{0}\right\}\right),\left(e_{2},\left\{\frac{u_{1}}{0.1}, \frac{u_{2}}{0}, \frac{u_{3}}{0.7}\right\}\right)\right\}
$$

is a $\left(v_{1}, v_{2}\right)$-g-closed fuzzy soft set.

We investigate some topological properties of a $\left(v_{1}, v_{2}\right)$-g-closed fuzzy soft set as in the following results.

Theorem 3.1. Let $v_{1}, v_{2}$ be two fuzzy soft topologies on $U$ such that $v_{2} \subset v_{1}$ and $\alpha_{A}, \beta_{A} \in F S S(U)_{E}$. If $\beta_{A} \sqsubseteq \alpha_{A} \sqsubseteq \widetilde{U}, \beta_{A}$ is a $\left(v_{1}, v_{2}\right)$-g-closed fuzzy soft set relative to $\alpha_{A}$ and $\alpha_{A}$ is a $\left(v_{1}, v_{2}\right)$-g-closed fuzzy soft set in $U$, then $\beta_{A}$ is $a\left(v_{1}, v_{2}\right)$-g-closed fuzzy soft set in $U$. 
Proof. Let $\beta_{A} \sqsubseteq \gamma_{A}$ and $\gamma_{A}$ be a fuzzy open soft set according to $v_{1}$. Then we get

$$
\beta_{A} \sqsubseteq \alpha_{A} \sqcap \gamma_{A}
$$

and

which follows that

$$
v_{\alpha_{A}}-F c l\left(\beta_{A}\right) \sqsubseteq \alpha_{A} \sqcap \gamma_{A},
$$

$$
\alpha_{A} \sqcap v_{2}-F c l\left(\beta_{A}\right) \sqsubseteq \alpha_{A} \sqcap \gamma_{A}
$$

and

$$
\alpha_{A} \sqsubseteq \gamma_{A} \sqcup\left[v_{2}-\operatorname{Fcl}\left(\beta_{A}\right)\right]^{c} .
$$

Since $\alpha_{A}$ is a $\left(v_{1}, v_{2}\right)$-g-closed fuzzy soft set and $v_{2} \subset v_{1}$, then we obtain

$$
v_{2}-F c l\left(\alpha_{A}\right) \sqsubseteq \gamma_{A} \sqcup\left[v_{2}-F c l\left(\beta_{A}\right)\right]^{c} .
$$

Hence we have

$$
v_{2}-\operatorname{Fcl}\left(\beta_{A}\right) \sqsubseteq v_{2}-\operatorname{Fcl}\left(\alpha_{A}\right) \sqsubseteq \gamma_{A} \sqcup\left[v_{2}-F c l\left(\beta_{A}\right)\right]^{c},
$$

whence

$$
v_{2}-\operatorname{Fcl}\left(\beta_{A}\right) \sqsubseteq \gamma_{A} .
$$

Consequently, $\beta_{A}$ is a $\left(v_{1}, v_{2}\right)$-g-closed fuzzy soft set in $U$.

Now we prove that the union of two $\left(v_{1}, v_{2}\right)$-g-closed fuzzy soft sets is a $\left(v_{1}, v_{2}\right)$-g-closed fuzzy soft set.

Theorem 3.2. Let $v_{1}, v_{2}$ be two fuzzy soft topologies on $U$ and $\alpha_{A}, \beta_{A} \in F S S(U)_{E}$. If $\alpha_{A}$ and $\beta_{A}$ are two $\left(v_{1}, v_{2}\right)$-g-closed fuzzy soft sets, then $\alpha_{A} \sqcup \beta_{A}$ is $\left(v_{1}, v_{2}\right)$-g-closed fuzzy soft.

Proof. If $\alpha_{A} \sqcup \beta_{A} \sqsubseteq \gamma_{A}$ and $\gamma_{A}$ is a fuzzy open soft set according to $v_{1}$, then we have

$$
v_{2}-\operatorname{Fcl}\left(\alpha_{A} \sqcup \beta_{A}\right)=v_{2}-F c l\left(\alpha_{A}\right) \sqcup v_{2}-F c l\left(\beta_{A}\right) \sqsubseteq \gamma_{A},
$$

since $\alpha_{A}$ and $\beta_{A}$ are two $\left(v_{1}, v_{2}\right)$-g-closed fuzzy soft sets. Therefore, $\alpha_{A} \sqcup \beta_{A}$ is $\left(v_{1}, v_{2}\right)$-g-closed fuzzy soft.

In the following example, we see that the intersection of two $\left(v_{1}, v_{2}\right)$-g-closed fuzzy soft sets is not always a $\left(v_{1}, v_{2}\right)$-g-closed fuzzy soft set.

Example 3.2. Let $U=\left\{u_{1}, u_{2}, u_{3}\right\}, E=\left\{e_{1}, e_{2}\right\}, v_{1}=\left\{\widetilde{\emptyset}, \widetilde{U}, \alpha_{E}\right\}$ and $v_{2}=\{\widetilde{\emptyset}, \widetilde{U}\}$ where $\alpha_{E}$ is a fuzzy soft set on $U$ defined as

Then the fuzzy soft sets

$$
\alpha_{E}=\left\{\left(e_{1},\left\{\frac{u_{1}}{0.3}, \frac{u_{2}}{0}, \frac{u_{3}}{0}\right\}\right),\left(e_{2},\left\{\frac{u_{1}}{0.3}, \frac{u_{2}}{0}, \frac{u_{3}}{0}\right\}\right)\right\} .
$$

$$
\beta_{E}=\left\{\left(e_{1},\left\{\frac{u_{1}}{0.3}, \frac{u_{2}}{0.4}, \frac{u_{3}}{0}\right\}\right),\left(e_{2},\left\{\frac{u_{1}}{0.3}, \frac{u_{2}}{0}, \frac{u_{3}}{0.5}\right\}\right)\right\}
$$

and

$$
\gamma_{E}=\left\{\left(e_{1},\left\{\frac{u_{1}}{0.3}, \frac{u_{2}}{0}, \frac{u_{3}}{0.7}\right\}\right),\left(e_{2},\left\{\frac{u_{1}}{0.3}, \frac{u_{2}}{0.6}, \frac{u_{3}}{0}\right\}\right)\right\}
$$

are two $\left(v_{1}, v_{2}\right)$-g-closed fuzzy soft sets. Also we obtain

$$
\beta_{E} \sqcap \gamma_{E}=\left\{\left(e_{1},\left\{\frac{u_{1}}{0.3}, \frac{u_{2}}{0}, \frac{u_{3}}{0}\right\}\right),\left(e_{2},\left\{\frac{u_{1}}{0.3}, \frac{u_{2}}{0}, \frac{u_{3}}{0}\right\}\right)\right\},
$$

which is not a $\left(v_{1}, v_{2}\right)$-g-closed fuzzy soft set.

Proposition 3.1. Let $v_{1}, v_{2}$ be two fuzzy soft topologies on $U$ such that $v_{2} \subset v_{1}$ and $\alpha_{A}, \beta_{A} \in F S S(U)_{E}$. Assume that $\alpha_{A}$ is a $\left(v_{1}, v_{2}\right)$-g-closed fuzzy soft set and $\beta_{A}$ is a fuzzy soft closed set relative to $v_{2}$. Then $\alpha_{A} \sqcap \beta_{A}$ is $a\left(v_{1}, v_{2}\right)$-g-closed fuzzy soft set.

Proof. Since $\beta_{A}$ is a fuzzy soft closed set relative to $v_{2}$, then $\alpha_{A} \sqcap \beta_{A}$ is a fuzzy soft closed set relative to $v_{2}$ in $\alpha_{A}$ whence it is $\left(v_{1}, v_{2}\right)$-g-closed fuzzy soft. By Theorem 3.1, $\alpha_{A} \sqcap \beta_{A}$ is a $\left(v_{1}, v_{2}\right)$-g-closed fuzzy soft set in $U$. 
We introduce the notion of the mixed $g$-fuzzy soft continuity in the following definition.

Definition 3.2. Let $U_{1}, U_{2}$ be two initial universe sets, $A, B \subseteq E$ two sets of parameters, $v_{1}, v_{2}$ two fuzzy soft topologies on $U_{1}$ and $v$ a fuzzy soft topology on $U_{2}$. Suppose that $u: U_{1} \rightarrow U_{2}, p: A \rightarrow B$ are two mappings and $f_{p u}: F S S\left(U_{1}\right)_{A} \rightarrow F S S\left(U_{2}\right)_{B}$ is a function. Then $f_{p u}$ is said to be mixed $g$-fuzzy soft continuous if $f_{p u}^{-1}\left(\beta_{A}\right)$ is a $\left(v_{1}, v_{2}\right)$-g-closed fuzzy soft set for every fuzzy soft closed set $\beta_{A}$ in $U_{2}$.

Finally, we prove a new version of the "Pasting Lemma" on a fuzzy soft topological space.

Theorem 3.3. (Pasting Lemma) Let $\widetilde{U}=\widetilde{U_{1}} \sqcup \widetilde{U_{2}}$ be a fuzzy soft topological space with two fuzzy soft topologies $v_{1}, v_{2}$ and $U_{3}$ a fuzzy soft topological space with a fuzzy soft topology $v$. Let $f_{p_{1} u_{1}}: F S S\left(U_{1}\right)_{A} \rightarrow F S S\left(U_{3}\right)_{B}$ and $f_{p_{2} u_{2}}: F S S\left(U_{2}\right)_{A} \rightarrow$ $F S S\left(U_{3}\right)_{B}$ be two mixed $g$-fuzzy soft continuous mappings where $p_{1}=p_{2}: A \rightarrow B, u_{1}: U_{1} \rightarrow U_{3}$ and $u_{2}: U_{2} \rightarrow U_{3}$ are functions. Suppose that $\widetilde{U_{1}}, \widetilde{U_{2}}$ are two $\left(v_{1}, v_{2}\right)$-g-closed fuzzy soft sets and $v_{2} \subset v_{1}$. If $u_{1}\left(u^{*}\right)=u_{2}\left(u^{*}\right)$ for every $u^{*} \in U_{1} \cap U_{2}$, then $f_{p_{1} u_{1}}$ and $f_{p_{2} u_{2}}$ combine to give a mixed g-fuzzy soft continuous mapping $f_{p u}: F S S(U)_{A} \rightarrow F S S\left(U_{3}\right)_{B}$ defined by the functions $p=p_{1}=p_{2}, u\left(u^{*}\right)=u_{1}\left(u^{*}\right)$ if $u^{*} \in U_{1}$ and $u\left(u^{*}\right)=u_{2}\left(u^{*}\right)$ if $u^{*} \in U_{2}$.

Proof. Let $\beta_{A}$ be a fuzzy soft closed set in $U_{3}$. Then we have

$$
f_{p u}^{-1}\left(\beta_{A}\right)=f_{p_{1} u_{1}}^{-1}\left(\beta_{A}\right) \sqcup f_{p_{2} u_{2}}^{-1}\left(\beta_{A}\right) .
$$

By the mixed $g$-fuzzy continuity of $f_{p_{1} u_{1}}$, we obtain that $f_{p_{1} u_{1}}^{-1}\left(\beta_{A}\right)$ is a $\left(v_{1}, v_{2}\right)$-g-closed fuzzy soft set in $U_{1}$. By Theorem 3.1, $f_{p_{1} u_{1}}^{-1}\left(\beta_{A}\right)$ is a $\left(v_{1}, v_{2}\right)$-g-closed fuzzy soft set in $U$ since $\widetilde{U_{1}}$ is $\left(v_{1}, v_{2}\right)$-g-closed fuzzy soft. Using the similar approach, we can easily see that $f_{p_{2} u_{2}}^{-1}\left(\beta_{A}\right)$ is a $\left(v_{1}, v_{2}\right)$-g-closed fuzzy soft set in $U$. By Theorem $3.2, f_{p u}^{-1}\left(\beta_{A}\right)$ is a $\left(v_{1}, v_{2}\right)$-g-closed fuzzy soft set in $U$. Consequently, $f_{p u}$ is a mixed $g$-fuzzy soft continuous mapping.

\section{Conclusion and Future Work}

In this paper, a new version of the pasting lemma are presented on a fuzzy soft topological space. To do this, we have used two fuzzy soft topological spaces. Similarly, the notion of a fuzzy soft Bitopological space was introduced with two fuzzy soft topological spaces [19]. Therefore, this problem can be considered on fuzzy soft Bitopological spaces using the concepts given in [19]. On the other hand, some applications of the pasting lemma can be investigated to analytic continuation on the complex plane as a future work.

Acknowledgements. The author would like to thank the anonymous referee for his/her comments that helped us improve this article.

\section{References}

[1] Açıkgöz, A., Taş, N.: Some new mixed soft sets. Mathematical Sciences and Applications E-Notes. 2 (2), 105-118 (2014).

[2] Açıkgöz, A., Taş, N. A., Noiri, T.: A decomposition of some types of mixed soft continuity in soft topological spaces. Filomat. 30 (2), 379-385 (2016).

[3] Ahmad, B., Kharal, A.: On fuzzy soft sets. Advances in Fuzzy Systems. 2009, 6 pages, Article ID 586507 (2009).

[4] Alcantud, J. C. R., Rambaud, S. C., Torrecillas, M. J. M.: Valuation fuzzy soft sets: a flexible fuzzy soft set based decision making procedure for the valuation of assets. Symmetry 9 (11), 253 (2017).

[5] Anitha, M., Selvi, R., Thangavelu, P.: Pasting lemmas for g-continuous functions. Missouri Journal of Mathematical Sciences. 21 (1), 28-33 (2009).

[6] Björn, A. Martio, O.: Pasting lemmas and characterizations of boundary regularity for quasiminimizers. Results in Mathematics. 55, 265-279 (2009).

[7] Császár, Á.: Mixed constructions for generalized topologies. Acta Mathematica Hungarica. 122 (1-2), 153-159 (2009).

[8] Demir, İ., Özbakır, O. B.: Some properties of fuzzy soft proximity spaces. The Scientific World Journal. 2015, 10 pages, Article ID 752634 (2015).

[9] Demir. İ., Özbakır, O. B.: An extension of Lowen's uniformity to the fuzzy soft sets. Konuralp Journal of Mathematics. 6 (2), 321-331 (2018). 
[10] Ergül, Z. G., Yüksel, Ş.: A new type of soft covering based rough sets applied to multicriteria group decision making for medical diagnosis. Mathematical Sciences and Applications E-Notes. 7 (1), 28-38 (2019).

[11] Gnanambal, Y., Balachandran, K.: On gpr-continuous functions in topological spaces. Indian Journal of Pure and Applied Mathematics. 30 (6), 581-593 (1999).

[12] İrkin, R., Özgür, N. Y., Taş, N.: Optimization of lactic acid bacteria viability using fuzzy soft set modelling. An International Journal of Optimization and Control: Theories and Applications (IJOCTA). 8 (2), 266-275 (2018).

[13] Kalaichelvi, Dr. A., Malini, P. H.: Application of fuzzy soft sets to investment decision making problem. International Journal of Mathematical Sciences and Applications. 1 (3), 1583-1586 (2011).

[14] Kannan, K., Rao, K. C.: Pasting lemmas for some continuous functions. Thai Journal of Mathematics. 12 (1), 245-249 (2014).

[15] Karaca, F., Taş, N.: Decision making problem for life and non-life insurances. Journal of Balıkesir University Institute of Science and Technology. 20 (1), 572-588 (2018).

[16] Mahanta, J., Das, P. K.: Results on fuzzy soft topological spaces. arXiv:1203.0634v1, (2012).

[17] Maji, P. K., Biswas, R., Roy, A. R.: Fuzzy soft sets. The Journal of Fuzzy Mathematics. 9 (3), 589-602 (2001).

[18] Molodtsov, D.: Soft set theory - first results. Computer Mathematics and its Applications. 37 (4-5), 19-31 (1999).

[19] Mukherjee, P., Park, C.: On fuzzy soft Bitopological spaces. Mathematics and Computer Sciences Journal. 10 (7), 1-8 (2015).

[20] Nazmul, S., Samanta, S. K.: Soft topological soft groups. Mathematical Sciences. 6 (1), 66 (2012).

[21] Özgür, N. Y., Taş, N.: A note on "application of fuzzy soft sets to investment decision making problem". Journal of New Theory 7, 1-10 (2015).

[22] Tanay, B., Kandemir, M. B.: Topological structure of fuzzy soft sets. Computer Mathematics and its Applications 61 (10), 2952-2957 (2011).

[23] Tas, N. A., Özbakır, O. B.: On some mixed types of continuity on generalized neighborhood systems. Journal of Advanced Studies in Topology. 5 (2), 32-43 (2014).

[24] Taş, N., Özgür, N. Y., Demir, P.: An application of soft set and fuzzy soft set theories to stock management, Süleyman Demirel University Journal of Natural and Applied Sciences. 21 (2), 791-196 (2017).

[25] Taş, N.: Two new versions of the pasting lemma via soft mixed structure (submitted).

[26] Tozlu, N., Yüksel, Ş.: Soft A-sets and soft B-sets in soft topological spaces. Mathematical Sciences and Applications E-Notes. 5 (2), 17-25 (2017).

[27] Varol, B. P., Aygün, H.: Fuzzy soft topology. Hacettepe Journal of Mathematics and Statistics. 41 (3), 407-419 (2012).

[28] Zadeh, L. A.: Fuzzy sets. Information and Control. 8, 338-353 (1965).

\section{Affiliations}

NIHAL TAŞ

AdDRESS: Balıkesir University, Dept. of Mathematics, 10145, Balıkesir-Turkey.

E-MAIL: nihaltas@balikesir.edu.tr

ORCID ID: http://orcid.org/0000-0002-4535-4019 